\title{
Neurociencias y psicoanálisis: consideraciones epistemológi- cas para una dialéctica posible sobre la subjetividad.
}

Neurosciences and psychoanalysis: epistemological considerations for a dialectical approach on subjectivity.

\section{Héctor García de Frutos ${ }^{a}$.}

${ }^{a}$ Departamento de Psicología Básica de la Universidad de Barcelona.

Correspondencia: Héctor García de Frutos (hectorgarcia@ub.edu)

Recibido: 29/03/2011; aceptado con modificaciones: 02/07/2011

\begin{abstract}
RESUMEN: Algunas aproximaciones contemporáneas de la subjetividad tratan de establecer puentes entre las neurociencias y el psicoanálisis. El presente trabajo comenta desde una perspectiva epistemológica las dificultades que estos enfoques presentan, argumentando que, si bien la subjetividad se puede pensar adecuadamente desde ambas disciplinas, se debe postular un concepto particular de subjetividad para cada una de ellas. Un diálogo entre neurociencias y psicoanálisis podría ser útil siempre que cada perspectiva se funde en una metodología sólida, empírica y apropiada desde el punto de vista epistemológico.

PALABRAS CLAVE: subjetividad, neurociencias, psicoanálisis, epistemología.
\end{abstract}

\begin{abstract}
Some contemporary approximations to subjectivity appeal to conceptual connections between neurosciences and psychoanalysis. The present work describes from an epistemological point of view the difficulties those approaches show, arguing that, even if subjectivity can be thought properly from both disciplines, each of them ought to do it separately and needs a particular concept to account for subjectivity. A dialogue between neurosciences and psychoanalysis could ultimately be useful if each perspective is founded in a solid, empirical and epistemologically appropriate methodology.

KEY WORDS: subjectivity, neurosciences, psychoanalysis, epistemology.
\end{abstract}

\section{Introducción: en busca del sujeto}

La subjetividad es hoy un tema candente para dos campos de estudio, las neurociencias y el psicoanálisis, que se han reencontrado estos últimos años.

El propósito del presente artículo es el siguiente: 1) discutir ciertas propuestas contemporáneas de interacción entre estas disciplinas; 2) argumentar que no se puede hablar con propiedad de la subjetividad al margen de una teoría sólida que defina y determine el concepto de sujeto del que estamos hablando. Esta teoría debe apoyarse en una metodología singular y rigurosa, que defina objetos de estudio coherentes desde el punto de vista epistemológico.

En consecuencia, veremos que a falta de una teorización que englobe a las neurociencias y al psicoanálisis, debe pensarse un concepto de subjetividad distinto para cada una de ellas. Mostraremos además que ambas disciplinas son epistemológicamente válidas, aunque presenten dificultades.

De estos postulados se sigue que se puede responder con rigor a la pregunta “¿quién causó la acción x?” al menos desde dos discursos, el neurocientífico y el 
psicoanalítico, siempre que admitamos que la persona se apropia adecuadamente de sus acciones al suponerlas suyas o subjetivas, o lo que es lo mismo, que la subjetividad juega un papel fundamental en la acción intencional de un agente. Un agente es, tradicionalmente, el individuo (o cosa) que produce un efecto; en el caso de una persona, el sujeto activo de una acción. Si la psicología popular sitúa a la persona en el origen de la acción intencional, las neurociencias y el psicoanálisis pueden situar al agente en otro lugar: en el primer caso, en la singularidad de la red neuronal del individuo considerado; en el segundo, en la configuración de creencias, fantasías y deseos inconscientes tras los que se postula, por razones éticas, un sujeto que elige.

Usualmente, se piensa que la subjetividad es la forma en que los individuos suponemos y accedemos a la propia identidad. No obstante, la investigación psicoanalítica permite observar una disyunción entre el sujeto y la constatación de la propia identidad, como veremos más adelante. La particularidad de este sujeto es que, siendo radicalmente distinto del yo (hablamos de un sujeto como operador mínimo producido por el lenguaje en tanto éste es un orden simbólico, mientras que el yo apunta a la identificación con la propia imagen, que es de otro orden), es a la vez la condición lógica previa a toda identificación yoica, siendo esta última, sin duda, la base de lo que llamamos identidad.

El desarrollo que llevaremos a cabo planteará además dos problemáticas para futuras reflexiones, a saber: (a) El problema de la agencia: El concepto tradicional de agente definido anteriormente no parece el más apropiado para un estudio riguroso de las causas de la acción; (b) El problema interdisciplinar: Si existen al menos dos formas diferentes pero epistemológicamente válidas de acceder a la subjetividad queda por saber si pueden conjugarse (y si es así, de qué forma) en una concepción interdisciplinaria más amplia.

\section{1) Algunos problemas de las neurociencias actuales...}

Resulta indudable: hay mente porque hay cerebro. No vamos a discutir este punto: primero, porque parece difícilmente discutible; y segundo, porque no es el propósito de este artículo. Las neurociencias son hoy una disciplina respetada, con un gran número de científicos trabajando en el mundo entero.

Uno de ellos, el premio Nobel Eric Kandel, sitúa la singularidad individual fundamental en la estructura cerebral de cada individuo y en sus particularidades mentales (1). Creo que podemos generalizar esta convicción a la mayor parte de científicos y pensadores contemporáneos: el cerebro es el órgano que constituye todas las particularidades mentales del sujeto. 
ORIGINALES Y REVISIONES

Por tanto, está justificado hablar de sujeto neurológico, ya que se admite casi universalmente que de la estructura neurológica depende la identidad mental del individuo, así como su singularidad para percibir, sentir, reflexionar, decidir, actuar, hablar, imaginar, o crear.

Sin embargo, no siempre es sencillo extraer conclusiones de las pistas que rastreamos en la actividad cerebral, especialmente si tratamos de ligar estas pistas con sistemas funcionales de la mente, o con la subjetividad (2). En efecto, si las investigaciones que apuntan a localizar funciones mentales en áreas cerebrales son numerosas, los resultados son en muchos casos contradictorios, como recuerda Coltheart (2): las técnicas de neuroimagen no establecen módulos por sí mismas, sino que pueden a lo sumo localizar módulos ya conceptualizados. Hay interdependencia entre el modelo preestablecido y el modelo que supuestamente estaría implícito en la organización cerebral: sólo se puede localizar lo que ya está conceptualizado.

Lo cierto es que la interpretación misma de los trabajos en neurociencias depende de determinados puntos de partida sobre el cerebro, de carácter teórico, algunos de los cuales vamos a considerar aquí. En muchos casos, se obvia la discusión de estos aspectos teóricos para llegar a conclusiones que parecen convincentes. Algunos serían:

1) El sistema nervioso central (SNC) se estudia usualmente como una unidad independiente. Pero no podemos olvidar que esto es un recorte conceptual. El SNC existe en conexión con órganos, glándulas, músculos $(3,4)$. No se limita al tejido intracraneal sino que incluye prolongaciones que permiten el funcionamiento del cuerpo entero de un individuo. Funciona por y en relación a estos tejidos, y necesita de estimulaciones adecuadas (tanto externas como internas), que hoy sabemos moduladas y de una gran complejidad (5). El argumento holista está muy manido, pero no por ello debe olvidarse. La neurociencia se basa generalmente en un recorte conceptual del SNC del resto de tejidos, y explicamos fenómenos en el cuerpo del ser humano a partir de su funcionamiento. Esta decisión no es gratuita: conlleva la construcción de un objeto ideal, un SNC estándar, universal, desligado del resto del organismo: el SNC del individuo normal.

2) Aunque hablamos del SNC, en la investigación empírica se asume y acepta ampliamente que la arquitectura neuronal se organiza en áreas (6): esto ha sido demostrado por ejemplo, de manera ampliamente aceptada y convincente, al aislarse un mapa neuronal del sistema de percepción visual. Sin embargo, estas áreas interactúan, lo que supone una configuración dinámica como ya propuso Luria (7). Por tanto, la investigación supone y trabaja generalmente desde una modularidad compleja, pero conceptualmente el SNC se piensa como una unidad. Surge inmediatamente una pregunta, a saber, si es pertinente hablar del "cerebro" o del SNC y, si lo es, explicar cómo y por qué mecanismos el cerebro "unifica" por así decirlo su 
actividad. Este es el problema de fondo que enfrenta hoy a los modelos modulares de la arquitectura neuronal con los modelos conexionistas.

3) El fenómeno de la plasticidad neuronal, demostrado a nivel neurofisiológico y cuya importancia conceptual no cesa de ir en aumento, impide concebir el cerebro como un objeto inmutable. Si a nivel macroscópico la anatomía permanece aparentemente inalterable, las conexiones celulares están siempre sometidas, al menos potencialmente, al cambio. Si somos rigurosos con los postulados de este fenómeno debemos admitir que si algo es válido para un cerebro hoy, no tiene por qué serlo el año que viene.

4) Tampoco podemos olvidar las exigencias que impone cualquier medición de la actividad cerebral: a saber, que el origen de una cadena de activaciones depende siempre de ciertos criterios metodológicos sujetos a discusión. En efecto, es la implantación (teórica y consensuada) de un tiempo cero y de una actividad base lo que marca el inicio de la medida y por tanto de la activación que da lugar a un fenómeno concreto. No en vano, Leibniz ya sostuvo que no es posible medir ni concebir una extensión sino a partir de puntos intensionales no sujetos a medición. Estos puntos intensionales vienen dados por la conciencia, e indudablemente, en el caso de la ciencia, por axiomas teóricos y acuerdos de la comunidad sobre la metodología de medida (8).

Algunos autores recuerdan apropiadamente ciertos aspectos de las técnicas de neuroimagen que exigen prudencia a la hora de interpretar los resultados que obtenemos con ellas. Por ejemplo:

Bub (9) recuerda entre otras cosas que en muchos estudios, para avalar ciertas hipótesis, se recurre a datos de la investigación clínico-anatómica, cuyas lesiones suelen ser muy poco específicas.

También señala que, en aquellos casos en que se mide experimentalmente las diferencias de activación entre dos grupos (control y experimental, en los que la tarea es idéntica pero difiere en un paso fundamental), las diferencias observadas pueden interpretarse al menos de dos maneras: a) Las zonas activadas en un grupo pero no en el otro dan cuenta de la tarea diferencial entre los grupos; o b) que estas diferencias de activación pueden dar cuenta de redes mucho más complejas en los que esta activación participa, sin que dicha zona dé cuenta exactamente de la tarea diferencial.

Por su parte, Logothetis (6) recuerda que las técnicas fMRI no muestran la activación neuronal directa sino que dependen del flujo de sangre, lo cual es problemático ya que lo que se mide es un subrogado que presenta problemas de especificidad espacial y de respuesta temporal. Esto limita la posibilidad de interpretación. Además, la técnica de fMRI no puede distinguir entre el procesamiento funcional específico y la neuromodulación, entre las señales neuronales de abajo-arriba o de arriba-abajo, y puede potencialmente confundir la excitación con la inhibición. 
ORIGINALES Y REVISIONES

Estas consideraciones apuntan a que el estudio del funcionamiento neural es muy complejo... Complejidad que no siempre se tiene en cuenta en la interpretación de los resultados de muchos estudios. Aún más compleja parece la demostración empírica de lo que parece una obviedad: que la configuración neuronal está a la base de la singularidad mental.

Sin embargo, las disciplinas neurocientíficas pueden enriquecerse ampliamente si tienen en cuenta la complejidad teórica que implica su trabajo, y buscan enriquecer dicha teoría mediante un serio trabajo epistemológico y conceptual.

Hemos visto por tanto que el SNC articula su funcionamiento al de otros órganos, que es una amplia red de interacciones de una gran complejidad, que responde de forma plástica a la experiencia, y del cual aún queda mucho por revelar. Sí, podemos hablar de subjetividad neuronal, pero aún queda mucho trabajo por hacer...

Veremos en el apartado 3 cómo algunos autores contemporáneos han abordado la subjetividad desde la neurociencia y apoyándose a la vez en las teorías psicoanalíticas, abriendo una nueva ola de intercambios que parecía impensable hace pocos años. Pero antes, examinemos como ha abordado el psicoanálisis la cuestión de la subjetividad.

\section{2) El sujeto del psicoanálisis.}

Y el psicoanálisis, ¿qué puede decirnos de la subjetividad? ¿Por qué un neurocientífico se interesaría por la disciplina acuñada por Freud?

La orientación que tomaremos para hablar de la práctica analítica es propiamente el viraje que el psiquiatra y psicoanalista Jacques Lacan imprimió a la lectura de los textos de Freud. Por supuesto, no es la única forma de abordar el psicoanálisis, pero la elegimos pues nos parece la más sólida en su cuestionamiento epistemológico, en su riqueza de referencias a otras disciplinas, así como en su capacidad para poner de relieve una ética que no es sino la de la subversión del sujeto.

Más allá de la teoría, el encuentro entre un analista y un paciente es un hecho. Ahí suceden cosas, y se constatan ciertos fenómenos. El psicoanálisis, contrariamente a lo que se piensa, es una disciplina empírica en tanto resalta lo que se dice, ni más ni menos. Esto nos parece válido para el psicoanálisis en su conjunto; por su parte, Lacan señala repetidas veces que el inconsciente es más lo que se escucha que el significado oculto tras las palabras que puede suponer un analista. Esto permite justificar que, lejos de toda hermenéutica, el método psicoanalítico se sostiene de actos de habla, fragmentos de discurso, los cuales se pueden considerar con total 
legitimidad como siendo empíricos. Por supuesto, lo que no implica en absoluto que sea posible hacer una muestra poblacional y tratar los datos de forma estadística: eso borraría absolutamente la dimensión del sujeto en juego.

Para constituir el conocimiento analítico se presentan casos clínicos uno por uno, con sus efectos, y se reporta muchas veces las frases tal y como el paciente las dice, siendo esta exigencia fundamental. En cualquier caso, el fenómeno de transferencia que se produce entre un paciente y su analista permite que el primero acceda de forma excepcional a algunos de sus contenidos mentales, de los que se sorprende o a los que responde con un afecto inesperado.

Quizás este dato sea el más fundamental para sostener la existencia de un inconsciente: el paciente dice cosas, aunque no sabe del todo lo que dice hasta que el analista le señala eso mismo que ha dicho. Nada nuevo al nivel del mensaje: lo que el paciente ha dicho ya lo conoce, puesto que lo ha dicho. Es escuchar a otro decir lo que uno ha dicho lo que revela algo nuevo, y ahí el cuerpo responde con la emoción. Algunos alegarán que esto se debe a la sugestión que el analista ejerce sobre el paciente, en tanto éste tiene la absurda convicción de que su analista sabe cosas sobre él que él mismo desconoce. Esto es fácilmente rebatible: en efecto, la interpretación del analista no siempre causa efectos ( ¿a qué se debería, entonces, que en algunos momentos los cause? Parecería que hay algo singular en esos dichos, por oposición a los otros que no generan afectos al ser señalados), y además al cabo de unos meses de análisis el paciente ya no le supone el saber al analista, sino a su propio inconsciente. Lo único que el analista sabe es escuchar el inconsciente, que no es otra cosa que la lógica que se repite en el discurso del paciente.

Si estos fenómenos se producen, y es un hecho que se producen (toda persona que se analiza o que se ha analizado puede testimoniar de estos momentos, que normalmente dan pistas sobre el propio análisis y suelen abrir una nueva ola de asociaciones o sueños), no vemos por qué no podría haber una explicación coherente de por qué sucede. Hasta hoy, ninguna disciplina que no sea el psicoanálisis ha explicado estos fenómenos; como es lógico, ya que el manejo del método que los posibilita es singular y se ha perfeccionado a lo largo de décadas.

La constatación que se extrae de la clínica analítica es sencilla: en el ser humano se establecen asociaciones entre los afectos y el discurso, y éste es el objeto privilegiado de estudio de la teoría psicoanalítica. A pesar de que la ciencia cognitiva empieza a interesarse estos últimos años por las emociones, la perspectiva del psicoanálisis es privilegiada pues maneja contenidos mentales que difícilmente se pueden conocer en un dispositivo que no permita una estrecha confidencialidad durante años. Sin desmerecer en absoluto los conocimientos de la ciencia cognitiva, el dispositivo analítico es único, y merece tenerse en cuenta no sólo como disciplina terapéutica, sino como lugar privilegiado de investigación del ser humano. 
ORIGINALES Y REVISIONES

Uno no desea lo que quiere, ni tiene por qué querer lo que desea: eso que el psicoanálisis llama inconsciente no es otra cosa que ciertas asociaciones lingüísticas y afectivas que configuran lo que desea una persona. Desear exige coherentemente un sujeto deseante, sujeto que se presenta como dividido en el psicoanálisis contemporáneo $(10,11)$. Fenomenológicamente, el individuo mismo se siente dividido ahí donde no cree haber escogido lo que desea. El psicoanálisis explicita esta división al distinguir el sujeto del yo. La persona que se presenta en sesión es el yo, la imagen de nosotros mismos que nos representa, con la que nos identificamos, y que modelamos a partir de rasgos que asumimos como propios. Es también el lugar de la voluntad consciente. Pero Freud (12) ya postulaba que gran parte del yo es inconsciente: en efecto, uno no siempre es consciente de las palabras o rasgos que lo conforman como persona. El psicoanálisis postula un sujeto, el sujeto del inconsciente, entre esas palabras, que deben tomarse en su valor significante según Lacan: es decir, la unidad simbólica mínima y diferenciada que invita a la significación, la cual no forzosamente es fija o unívoca. Es un sujeto que escoge en determinado momento algunas, y no otras, para representarse. Recordemos que esto no es una construcción teórica de la subjetividad fruto de la reflexión, sino una explicación coherente de lo que sucede en un psicoanálisis: una persona entra, habla de sí, se sorprende al escucharse decir ciertas palabras y creencias que suponía inmutables pero jamás se planteó y, a veces, ve como tales palabras pierden su función, su peso, su valor. Y es por ello que puede (y podemos, en general) argumentar que estos rasgos, fundamentalmente lingüísticos, son una elección inconsciente: uno no sabe que los eligió hasta que un día, hablando, estos dejan de valer lo que valían, y se abre la posibilidad de reemplazarlos por otros, más vivibles.

Además, no se puede excluir del psicoanálisis una posición ética, según la cual el sujeto es responsable. Responsable en gran medida de lo que le sucede, responsable totalmente de lo que ha elegido. Sin embargo, es preciso mantener presente la distinción entre sujeto y yo para saber que, aunque uno es responsable, no siempre lo sabe, y es en análisis dónde puede discernir en qué momento una elección suya le llevó allí donde se encuentra ahora.

En definitiva: sí, el psicoanálisis es una forma de explorar la subjetividad, como muestran todas y cada una de las presentaciones de casos que se realizan, y de las que existe una extensa bibliografía. Y sí, es una disciplina epistemológicamente válida en tanto:

1) El paciente considera esos contenidos no solo como propios, sino como esencialmente constituyentes de su singularidad, ¿y quién sino él podría emitir algún juicio de veracidad sobre sus propios contenidos mentales?

2) El analista se limita a subrayar las palabras que dice el paciente, y es solo desde estas palabras desde donde construye la lógica del caso. 
3) Las intervenciones del analista tienen a veces efectos subjetivos y afectivos que cambian rasgos, sentimientos o comportamientos del paciente, y en este sentido una cura analítica es efectiva.

4) Es a partir de los casos, única y exclusivamente, desde donde se construye la teoría, siempre en construcción y abierta a revisión.

Sin embargo, no podemos olvidar las dificultades epistemológicas de la teoría analítica: en efecto, en tanto trabaja desde la transferencia, y fundándose en la subjetividad, sus resultados no se pueden objetivar, ni se pueden establecer leyes causales sobre los fenómenos que reporta. No puede por tanto ser una disciplina científica, y esto para algunos cuestiona su valor $(13,14,15)$.

\section{3) ¿Pueden las neurociencias rescatar al psicoanálisis?}

Algunos autores valoran el psicoanálisis como teoría, pero admiten que no puede ser una ciencia. Para solventar el problema interdisciplinar que adelantábamos en la introducción y las dificultades epistemológicas del psicoanálisis, proponen que éste se base en los datos de la neurociencia para reformular su teoría y hacerla científica. Kandel es el principal exponente de esta idea.

En efecto, para Kandel $(16,17)$, (a) el psicoanálisis representa la visión más coherente e intelectualmente satisfactoria de la mente. Pero, sugiere, (b) no ha evolucionado científicamente, y ya nada más se puede extraer de la escucha de los pacientes. Habría que seguir las indicaciones de Freud $(18,19)$ : será un día posible basar la teoría psicoanalítica en la biología. Además, (c) las neurociencias cognitivas son hoy la ciencia paradigmática para estudiar el cerebro.

De estas tres proposiciones se deduce una solución óptima: que el psicoanálisis reconstituya su teoría, su clínica y su práctica basándose en las neurociencias, y que a la vez provea a éstas de las herramientas conceptuales necesarias para que su concepción de la mente sea lo suficientemente amplia y compleja. Es decir: de la unión entre psicoanálisis y neurociencia puede surgir el más coherente y completo estudio de la subjetividad.

Para esta unión se exige una naturalización del psicoanálisis: Kandel sugiere que el conocimiento psicoanalítico debe estar sometido a vigilancia por parte de un observador externo, ya que los datos psicoanalíticos solo provienen de la visión subjetiva del psicoanalista. Es decir, someter el psicoanálisis al método científico, a la objetividad. Se puede así leer entre líneas una convicción de Kandel: de la escucha de una persona en el contexto analítico no se puede extraer ninguna verdad sostenible, ya que no es objetiva. 
ORIGINALES Y REVISIONES

Kandel cae en una primera contradicción: a la vez que sostiene que el psicoanálisis tiene el mejor modelo de la mente posible (curiosamente, ninguna argumentación apoya esta idea), olvida que la teoría psicoanalítica se apoya en lo que Freud llamó una metapsicología, es decir, una conceptualización teórica en la que Freud (20) no creía demasiado: "Uno no puede menos que decirse: "Entonces es preciso que intervenga la bruja». La bruja metapsicología, quiere decir. Sin un especular y un teorizar metapsicológicos - a punto estuve de decir: fantasear- no se da aquí un solo paso adelante".

En efecto, la metapsicología existe no por sí misma, sino para dar un marco teórico a la clínica, estando siempre supeditada a ella. La clínica psicoanalítica, independientemente de la escuela en que se base la práctica, exige la exploración de la singularidad mental de cada analizante a partir de la transferencia, que necesita una absoluta confidencialidad e intimidad entre el analista y el paciente. La inclusión de un elemento de evaluación objetiva es contraria al psicoanálisis porque imposibilitaría la confidencialidad e iría contra la ética y la teoría psicoanalítica.

Si la teoría psicoanalítica se funda en la clínica de la escucha, pero nada nuevo (¿por qué? Kandel no lo argumenta) ni nada verdadero (puesto que requiere objetivación) se puede obtener de dicha escucha, ¿Cómo es posible que el psicoanálisis haya dado con el mejor modelo de la mente posible? Esto sería parecido a decir que la astrología ha formulado el mejor modelo posible del sistema solar. En efecto, de un método falaz (puesto que para dar lugar a alguna verdad se requeriría de observadores externos, y no se ha hecho) y obsoleto (puesto que ya no puede decir nada nuevo), ¿cómo podría surgir un modelo conceptual adecuado?

Un segundo problema grave que plantean las ideas de Kandel refiere también a un deseo de ignorancia de la realidad de la clínica psicoanalítica: en un psicoanálisis, se habla. Nada más, pero nada menos. En ese sentido, la insistencia de Lacan en prestar atención al lenguaje es especialmente comprensible.

Que el psicoanálisis se funde en la neurociencia, es una idea errónea en tanto el discurso del paciente es aquello justamente que se analiza, y este se funda en el fenómeno de la intersubjetividad, de la creencia y de la interpretación, como sostiene Cavell (10) desde una óptica distinta a la lacaniana. Hoy, la neurociencia no tiene las herramientas para fundar un saber sobre la interpretación del discurso intersubjetivo, ni para localizar la sede de las creencias íntimas de un sujeto y su articulación. El día en que se puedan explicar los mecanismos neurofisiológicos y neuroanatómicos de la interpretación y la creencia, y observar directamente las modificaciones en las redes neuronales en que se basan y que causan estos fenómenos subjetivos, entonces se podrá explicar en términos biológicos lo que sucede en el cerebro durante una terapia analítica. Ese día no ha llegado, y por tanto lo más fundamental de la clínica psicoanalítica no tiene aún su correlato en las medidas neurocientíficas. 
Este segundo problema afecta especialmente a la obra de Solms y Turnbull (21), trabajo en que los autores apuestan por una síntesis de las perspectivas psicoanalítica y neurocientífica para cernir la subjetividad, proponiendo una nueva disciplina: el neuropsicoanálisis. En efecto, en su libro proponen que la investigación de pacientes con lesiones cerebrales mediante psicoterapia puede no sólo refundar la teoría psicoanalítica, sino sentar las bases para una adecuada arquitectura de la mente (Su método de investigación se describe en particular en el capítulo octavo del libro, en referencia a un estudio que realizaron Kaplan-Solms y Solms en el año 2000 sobre la relación entre el sistema inconsciente postulado por Freud y el hemisferio derecho).

Pero parece complicado, sino imposible, correlacionar adecuadamente la lesión anatómica macroscópica común a una cierta cantidad de sujetos, esto es, un hecho biológico estandarizado, con la singularidad de un discurso, interpretado además por un analista (la concepción de Solms y Turnbull sobre el análisis sostiene que es un proceso introspectivo; obviamente, viene a la mente una sencilla pregunta: ¿Si uno accede a sus contenidos mentales de forma única y privilegiada por introspección, para qué se necesita a un analista?).

En definitiva, el salto epistémico parece, cuanto menos, arriesgado...

\section{4) El neuropsicoanálisis frente a Davidson y el argumento del monismo anómalo.}

Una dificultad epistemológica para que las neurociencias contemporáneas den cuenta adecuadamente de la subjetividad tiene que ver justamente con la exigencia de objetividad y universalidad que toda ciencia requiere, y con la forma de estudio que eso conlleva en la investigación. Se establecen teorías y estructuras de funcionamiento a partir de muestras de sujetos (así se procede, sin ir más lejos, en el método de Solms y Turnbull). Por ejemplo, los trabajos científicos basados en las técnicas de neuroimagen suelen presentar las imágenes que se obtienen de superponer fotografías de cerebros de varios sujetos. Esto conlleva la suposición de un SNC ideal, del que sería el ser humano normal, como señalamos anteriormente. Esto sucede especialmente en los estudios de psicopatología: se habla del cerebro del esquizofrénico, del depresivo, o del autista. Sin embargo, el trastorno mental se diagnostica hoy mediante criterios normativos estándar fruto de una exploración psicológica a partir de manuales exclusivamente clasificatorios, como el DSM IV-TR (22). Sólo a partir de ahí se investigan los correlatos neuronales de dichos trastornos. No se nos puede escapar que, en el origen de la investigación, se asume una clasificación consensuada, pero que se define como ateórica y que se desentiende de la dimensión causal. La validez científica de esta metodología es 
ORIGINALES Y REVISIONES

más que cuestionable, en tanto la ciencia trabaja siempre desde modelos teóricos para deducir leyes de causalidad o correlaciones explicativas. Sólo hay cerebro del esquizofrénico porque suponemos un individuo esquizofrénico estándar.

Entonces, ¿qué nos puede decir hoy la neurociencia de la singularidad del cerebro de cada individuo? Opinamos que hasta ahora poca cosa, pero esto no implica que no pueda cambiar en el futuro. La única forma posible de aprehender al sujeto neuronal vendría de estudios extensos (neurofisiológicos, neuroanatómicos, mediante neuroimagen...) que examinen las particularidades cerebrales de un único caso.

En cuanto a la relación con el sujeto psíquico, se podría pensar, con Solms y Turnbull, que estos estudios permitirán relacionar singularidades en la estructura cerebral a singularidades en el comportamiento y el funcionamiento mental, cerrando quizás así, algún día, la brecha del problema mente-cuerpo (23)...

Y sin embargo, aún en el caso de que un día estos estudios adquirieran el nivel de perfección suficiente como para poder explicar la totalidad de las causas físicas a la base de cualquier acción, persistiría un problema epistemológico. Es el problema que señala brillantemente Donald Davidson (8) y que da lugar a la teoría del monismo anómalo.

Davidson postula que, a nivel ontológico, un acontecimiento psíquico es siempre un acontecimiento físico. En ese sentido su teoría es monista, es decir, que niega la existencia del campo mental al margen de un campo físico (neuronal) que lo determina. Sin embargo, esta relación solo puede darse para acontecimientos psíquicos puntuales, fechados, esto es, tomados uno a uno; esta relación de identidad no puede darse en forma de leyes psicofísicas para tipos de acontecimientos mentales. ¿Por qué?

Porque la forma de definir un acontecimiento psíquico es que es un acontecimiento que implica para su descripción el uso de términos mentales, como las actitudes proposicionales (de la misma forma que un acontecimiento físico es cualquier acontecimiento que se pueda describir mediante términos físicos).

Los términos mentales incluyen al menos un verbo del tipo creer, recordar, sentir, percibir... verbos que remiten a la característica de intencionalidad que postulaba Brentano. Son expresiones características del lenguaje humano, siendo éste un sistema abierto a la interpretación que no admite leyes estrictas que relacionen unos acontecimientos con otros: no se puede nunca predecir (aunque se pueda suponer, o determinar estadísticamente su probabilidad de ocurrencia) qué acontecimiento mental seguirá a un acontecimiento mental dado.

Los acontecimientos mentales se expresan como deseos, creencias, intenciones, recuerdos, y en definitiva determinan (al menos en parte) cualquier acción humana que se considere conducta y no sea una respuesta instintiva, siempre que para su descripción se empleen términos psicológicos. 
Por tanto, no se pueden establecer leyes psicofísicas en sentido estricto, sino únicamente generalizaciones (siempre variables) a partir de estadísticas, ya que lo psíquico viene determinado por un sistema abierto y lo físico tiene, por definición, una extensión cerrada.

En tanto consideremos que la singularidad del ser humano puede definirse adecuadamente por actitudes proposicionales que expresan creencias, deseos, afectos, recuerdos, motivaciones, intenciones, decisiones..., y por muy extensa que sea la descripción física de las bases neuronales del comportamiento humano, tendremos siempre dos formas epistemológicamente válidas de dar cuenta de los mismos eventos. La descripción psíquica no podrá ser reducida a las explicaciones físicas si aceptamos como válida la idea de Davidson: que las razones de una persona pueden ser consideradas causas de sus actos, y que dichas razones incluyen siempre potencialmente cierta cuantía de indeterminación.

\section{5) La plasticidad neuronal: la subjetividad emerge en las neurociencias.}

Si hay algún fenómeno biológico que permite pensar la indeterminación, la decisión, la libertad o la interpretación, este sería la plasticidad neuronal. Esta realidad impide pensar el cerebro como un sistema cerrado sometido a leyes, introduciendo en la ecuación los conceptos de contingencia y de mutabilidad. Para Ansermet y Magistretti (24), psicoanalista y neurobiólogo respectivamente, el fenómeno de la plasticidad neuronal constituye por sí mismo un nuevo paradigma que entierra el determinismo genético y los modelos de interacción genética/ambiente. Se apoyan para ello en el siguiente argumento: si hoy sabemos como se inscribe la experiencia en el sistema neuronal, y sabemos que dicha inscripción determina al sujeto, debemos aceptar que entre la genética y el ambiente interviene la plasticidad neuronal, una determinación constituida por las vivencias singulares que vienen a inscribirse en una red que se reconfigura constantemente. La subjetividad se torna así una noción central tanto para la neurociencia como para el psicoanálisis.

Los autores plantean la similitud de esta problemática con la que introduce el descubrimiento de la epigenética, fenómeno que permite relacionar los rasgos del ambiente con el genoma. En efecto, la epigenética determina qué causas ambientales influyen en que algunos genes se expresen en determinado momento cuando hasta ese momento no lo habían hecho. Este nuevo campo de investigación da lugar a una ruptura del debate sobre la existencia o no de un determinismo genético estricto.

$\mathrm{Su}$ obra recorre la teoría psicoanalítica y los últimos hallazgos en neurofisiología para postular lo siguiente: 1) la huella no sólo se inscribe una vez, sino que lo hace en sucesivas reescrituras a distintos niveles (consciente e inconsciente, 
ORIGINALES Y REVISIONES

como mínimo), reconfigurando la experiencia hasta que ésta queda desdibujada. Esta suposición teórica ya la albergó Freud (18). La subjetividad reside en estas reescrituras sucesivas. 2) Se puede tender un puente entre huella psíquica y huella sináptica, teniendo también en cuenta que esta última se relaciona siempre con un estado afectivo de tipo somático.

Hay al menos dos formas de interpretar las tesis de Ansermet y Magistretti:

a) la forma débil: el modelo neurobiológico de inscripción de la huella puede ilustrar la biología de la formación del inconsciente y abrir una vía de diálogo sobre la subjetividad entre neurociencias y psicoanálisis, a partir de la plasticidad.

b) la forma fuerte: el sujeto psicoanalítico, cuya sede es el inconsciente, es equivalente al sujeto neurológico (o lo que es lo mismo, las singularidades de las redes neuronales). En esta perspectiva, la relación entre la huella y el estado somático da cuenta de la articulación entre el sujeto y su objeto de deseo.

La distinción es clara: en un caso las vías epistemológicas son netamente distintas y dan fe de niveles de realidad distintos no reducibles el uno al otro. En el otro, la plasticidad neuronal permite una naturalización adecuada de la teoría analítica al dar cuenta de la inscripción de la experiencia, y por tanto podría un día sostener el andamiaje psicoanalítico.

Los autores defienden que hay un punto de intersección (la inscripción de la experiencia a partir de la plasticidad neuronal) entre psicoanálisis y neurociencia, aunque mantienen que no hay síntesis posible entre estos dos campos. ¿Qué los diferencia? Los autores nadan en la vaguedad conceptual al sostener lo siguiente: “AAcaso el sujeto de las neurociencias y del psicoanálisis no es el mismo?”.

De la misma forma, a la vez que localizan al sujeto en la plasticidad neuronal, afirman que la inscripción parcial y sucesiva de la experiencia aleja al sujeto de la experiencia misma. Es decir, que el sujeto existe gracias a la plasticidad neuronal, pero la misma plasticidad aleja al sujeto de la experiencia. ¿Cuántos sujetos hay en juego?

Este es el verdadero problema de la tesis de Ansermet y Magistretti (24): al naturalizar el significante identificándolo a la huella sináptica no se ve por qué psicoanálisis y neurociencia no estarían en definitiva estudiando el mismo objeto, lo que implicaría que ambas teorías compiten para hallar la mejor explicación posible de la subjetividad. En este caso, puesto que el objeto de estudio es en último término la plasticidad neuronal, la neurociencia parecería la disciplina más apropiada...

Pero al tratar de preservar lo mejor de cada disciplina en una teoría conjunta, no pueden evitar mantener un dualismo subjetivo, en el que la red neuronal formada por plasticidad es y no es el sujeto que acude al diván. Postular este punto de intersección les lleva a una confusión terminológica.

El sujeto de las neurociencias y del psicoanálisis no es el mismo porque en un caso es material, observable, y está sometido a leyes biológicas (todo esto es 
cierto para la plasticidad neuronal), y en el otro se trata de una suposición ética, fruto de la subjetivación del lenguaje, y que supone para hablar de ello un sistema intersubjetivo, interpretativo y abierto a la posibilidad de decidir. Si dos conceptos no se conocen ni se describen de la misma manera no pueden dar cuenta de lo mismo. En efecto, al sujeto psicoanalítico se le supone la responsabilidad de sus actos; se le supone no ya como siendo autor de su decisión, sino como el lugar en que la decisión ex-nihilo es posible. Es un sujeto intencional. La red neuronal, si bien es plástica y compleja, no es intencional.

No podemos pensar al fenómeno de plasticidad neuronal como un sujeto responsable, puesto que se basa en procesos fisiológicos no intencionales, ni como lugar de la decisión ex - nihilo, puesto que por definición viene dado en una red de contingencias ya establecida.

\section{6) No hay síntesis posible: un sujeto y un objeto de estudio para cada disciplina.}

Podemos oponer a las formulaciones de Ansermet y Magistretti las de E. Laurent (25), y de Bassols, Berenguer y Laurent (26): estos autores aluden a dos aspectos de la teoría psicoanalítica (el sujeto y el objeto) para mantener que psicoanálisis y neurociencia tratan de aprehender reales diferentes.

a) el sujeto: para Laurent (26) el sujeto del psicoanálisis es más que intersubjetivo; se forma a partir de un código (el lenguaje, lugar externo impuesto a los hablantes) en un contexto, por identificación. Se construye entre un sujeto (podemos imaginar a un niño dirigiéndose a un adulto que le enseña a hablar) y su interlocutor (el adulto) en una dialéctica. El punto crucial de Laurent a este respecto es señalar la existencia de un lapso, un punto de vacío en este intercambio: el sujeto se pregunta por lo que quiere el Otro de él, no comprende por qué el Otro le habla, o no acierta a comprender lo que el Otro le transmite. Este punto de vacío imposibilita la comunicación, introduciendo la dimensión del equívoco, así como la del deseo; es un vacío constitutivo del lenguaje humano en tanto éste nos sirve en efecto para abstraer, o hablar de una cosa en su ausencia. El código es inevitablemente abierto.

Es preciso quizás recordar en este punto que el término "Otro" es tomado de la obra de Jacques Lacan. Tiene múltiples lecturas en función del momento de evolución teórica. Aquí, entendemos por Otro al interlocutor al que uno le supone un saber, y que se presenta ante nosotros como extraño o poco comprensible, siendo difícil que uno lo vea como un igual. El ejemplo más sencillo es la forma en que un niño se representa a sus progenitores, pudiendo encarnar ambos a este Otro.

¿Qué hace que el sujeto entienda, dé significado a las palabras del Otro, y se represente mediante algunas de ellas? Para el psicoanálisis la constitución subje- 
ORIGINALES Y REVISIONES

tiva se establece en un eje donde la significación (apuntar a un significado) viene del Otro y el significado queda fijado por el propio afecto en el cuerpo. La clínica psicoanalítica encuentra sus puntos de dificultad en enunciados dolorosos, interpretados singularmente por el sujeto. Es decir, que el sujeto no sólo decide y se presenta mediante ciertas palabras, sino que da significado a éstas en función del afecto a ellas asociado. En efecto, esto se hace presente en la sesión analítica cuando, mientras se habla de algunas de las palabras que identifican al sujeto y se cuestiona su significación en un contexto vivido, estas palabras pierden su valor afectivo, y surge así una sensación de vacío y un nuevo afecto, normalmente el de la angustia. Este momento de tránsito es crucial para que el sujeto posteriormente pueda identificarse a nuevos enunciados menos sintomáticos.

El sujeto de las neurociencias no puede postular lógicamente el lugar del vacío en tanto, al menos a día de hoy, se preocupa por la observación de la actividad de las redes neuronales, que siempre están ahí, en el cerebro. Se deduce que es imposible identificar lo uno a lo otro.

b) el objeto: Bassols, Berenguer y Laurent (26) acuerdan que si las neurociencias requieren criterios de objetividad, el psicoanálisis se funda en criterios de "objetalidad". Es decir, el real por el que se interesa el psicoanálisis es no sólo el real del afecto, en tanto es sentido en un cuerpo, sino el de las coordenadas fantasmáticas en las que se presenta. Estas coordenadas organizan un escenario inconsciente en el que el sujeto se ubica, unas veces en un rol, otras en otro: puede observarse en el ejemplo princeps de escenario fantasmático que es 'pegan a un niño' (27). Son coordenadas tanto imaginarias (se presentan en imágenes) como significantes (se acompañan de enunciados). Este fantasma es la forma de presentación que determina para el sujeto su objeto de deseo, y constituye el núcleo del inconsciente. Por su parte, las neurociencias tienen un objeto de estudio, el cerebro, que nada tiene que ver con éste, en tanto es un objeto físico, un órgano al que se accede de forma consciente.

Si pensamos el psicoanálisis como un método de investigación de la mente humana, debemos saber por tanto que: 1) su objeto no es físico, sino psíquico; 2) $\mathrm{Su}$ objeto no es accesible a la conciencia, sino que se deduce del trabajo analítico que opera por desciframiento del inconsciente; 3) Su objeto puede considerarse rigurosamente en tanto su reconstrucción mediante análisis tiene efectos subjetivos notorios en la vida de los pacientes y en su realidad social. Uno y otro objeto no tienen por tanto nada que ver. 
Conclusión: una invitación al diálogo, no a la síntesis.

La neurociencia debería tener en cuenta el concepto de sujeto neurológico, ya que ha mostrado experimentalmente y explicado funcionalmente parte de los mecanismos a la base de los fenómenos de la plasticidad neuronal. Por tanto, es pertinente y posible pensar la subjetividad en neurociencias.

Sin embargo, la metodología neurocientífica presenta un sesgo: se elaboran muestras de sujetos que permiten la interpretación de leyes de funcionamiento a partir de correlaciones de tipo probabilista. Si bien esta es una forma adecuada de proceder para generalizar determinados mecanismos (por ejemplo, la percepción visual humana, o el procesamiento de estímulos auditivos), esta forma no es adecuada para el estudio de la singularidad subjetiva que nos presenta la plasticidad neuronal.

Puede haber actualmente otro problema: si bien las técnicas permiten hoy determinar ciertos mecanismos generales, no se conocen aún adecuadamente los mecanismos neuronales a la base de la intersubjetividad, la creencia, la interpretación, las condiciones de amor o la toma de decisiones. En ese sentido, la neurociencia no tiene aún una explicación adecuada de lo que sucede en la clínica psicoanalítica. Por estas dos razones, entre otras, la complementación que propone Kernberg (28) en la línea ideológica de las propuestas de Kandel (16), entre el campo de la investigación empírico-estadística, la investigación conceptual y la praxis clínica nos parece osada y de difícil justificación en cuanto a sus premisas epistemológicas. No hay sujeto en la muestra estadística, pues de cada uno se toma un significante que lo representa como idéntico al de otro sujeto de la muestra. El sujeto sólo se manifiesta como objeto de estudio. La singularidad se descuida, y sin embargo ésta es propiamente lo que pone de manifiesto la clínica: no hay el sujeto normal, depresivo o esquizofrénico. Hay una disyunción entre el sujeto que aísla la praxis analítica y el significante que lo representa. Entre ambos, es la dimensión del deseo la que está en juego, dimensión que orienta la cura analítica, al menos desde Lacan.

A lo sumo, el estudio de la plasticidad neuronal es sin duda la vía adecuada para investigar la inscripción de la experiencia singular en la red neuronal, pero poder dar el salto de la singularidad física a la subjetividad mental parece aún una meta muy lejana, epistemológicamente imposible si aceptamos el argumento de Davidson.

Por su parte, el psicoanálisis postula la subjetividad de una forma muy distinta: es una suposición ética que va siempre articulada a una dimensión afectiva. Es justamente en este punto en el que resulta imposible ligar este concepto al de plasticidad neuronal, pues este es un hecho que no requiere de ninguna ética. Pero suponer al sujeto en psicoanálisis no es sólo ético: tiene también resultados clínicos de incalculable valor terapéutico. 
El psicoanálisis, por su forma de trabajo, se intuye como una disciplina que tiene mucho que decir aún sobre la subjetividad mental, pero no se puede hacer una síntesis de las neurociencias y del psicoanálisis si tenemos en cuenta los argumentos que hemos presentado en el presente trabajo. Son disciplinas distintas, con metodologías particulares, y que investigan dominios que conocemos de formas diferentes: lo mental y lo físico. Cada una de ellas debe poseer su propio concepto de subjetividad. El interés de que el conocimiento sea fragmentario es justamente que se pueden establecer diálogos fructíferos entre sus distintas formas, siempre que se acepte la singularidad de cada campo, y respetando que cada disciplina tiene una verdad que comunicar. Esta parece la vía más respetuosa y la más válida.

\section{BIBLIOGRAFÍA:}

(1) Kandel E. Genes, brains and self-understanding; en Psychiatry, psychoanalysis, and the new biology of mind. Arlington, VA: American Psychiatric Publishing Inc, 2005.

(2) Coltheart M. Brain imaging, connectionism and cognitive neuropsychology. Cognitive Neuropsychology 2004; 21-1: 21-25.

(3) Dewey F, Freeman J, Froelicher V, Hadley D, Myers J. (2006). Autonomic nervous system interaction with the cardiovascular system during exercise. Progress in Cardiovascular Diseases 2006; 48-5: 342-362.

(4) García Segura L M. Hormones and brain plasticity. NY: Oxford University Press, 2009.

(5) Changeux J P, Christen Y, Damasio A, Singer W. Neurobiology of human values. Berlin Heidelberg: Springer-Verlag, 2005.

(6) Logothetis N. What we can do and what we cannot do with fMRI. Nature 2008; 453: 869878.

(7) Luria A R. El cerebro en acción. Barcelona: Fontanella, 1979.

(8) Davidson D. Filosofía de la psicología. Barcelona: Anthropos, 1994.

(9) Bub D. Methodological issues confronting PET and fMRI studies of cognitive function. Cognitive Neuropsychology 2000; 17-5: 467-484.

(10) Cavell M. The psychoanalytic mind: from Freud to philosophy. Cambridge, MA: Harvard University Press, 1993.

(11) Lacan J. La significación del falo; en Escritos (vol. 2). Buenos Aires: Siglo XXI, 2002.

(12) Freud S. El yo y el ello; en Obras completas de Sigmund Freud (vol. XIX). Buenos Aires: Amorrortu, 1992.

(13) Popper K. Conjectures and refutations: the growth of scientific knowledge. London: Routledge \& Kegan Paul, 1963.

(14) Grunbaum A. The Foundations of psychoanalysis: a philosophical critique. Berkeley, CA: University of California Press, 1984.

(15) Ardila R, Bunge M. Filosofía de la psicología. México: Siglo XXI, 2002.

(16) Kandel E. A new intellectual framework for psychiatry; en Psychiatry, psychoanalysis, and the new biology of mind. Arlington, VA: American Psychiatric Publishing Inc, 2005. 
(17) Kandel E. Biology and the future of psychoanalysis; en Psychiatry, psychoanalysis, and the new biology of mind. Arlington, VA: American Psychiatric Publishing Inc, 2005.

(18) Freud S. Proyecto de una psicología para neurólogos y otros escritos. Madrid: Alianza, 1974.

(19) Freud S. Más allá del principio del placer; en Obras completas de Sigmund Freud (vol. XVIII). Buenos Aires: Amorrortu, 1992.

(20) Freud S. Análisis terminable e interminable; en Obras completas de Sigmund Freud (vol. XXIII). Buenos Aires: Amorrortu, 1991.

(21) Solms M, Turnbull O. El cerebro y el mundo interior. México: FCE, 2003.

(22) López-Ibor Aliño, J J, Valdés Miyar M. DSM-IV-TR: Manual diagnóstico y estadístico de los trastornos mentales, texto revisado. Barcelona: Masson cop, 2002.

(23) Amstrong D M. The mind-body problem: an opinated introduction. Boulder, CO: Westview press, 1999.

(24) Ansermet F, Magistretti P. A cada cual su cerebro: plasticidad neuronal e inconsciente. Madrid: Katz, 2006.

(25) Laurent E. Lost in cognition: el lugar de la pérdida en la cognición Buenos Aires: Colección Diva, 2005.

(26) Bassols M, Berenguer, E, Laurent, E. Lost in Cognition. Freudiana 2005; 46: 145-167.

(27) Freud S. «Pegan a un niño». Contribución al conocimiento de la génesis de las perversiones sexuales; en Obras completas de Sigmund Freud (vol. XVII). Buenos Aires: Amorrortu, 1992.

(28) Kernberg O. The Pressing Need to Increase Research in and on Psychoanalysis. International Journal of Psychoanalysis 2006; 87: 919-926. 